Current Topics: Current

Status and Future Perspective

on the Application of Host

Defense Peptides to Medicine

Juntendo Medical Journal

2016. $62(2), 98-104$

\title{
Modulation of Macrophage Cell Death, Pyroptosis by Host Defense Peptide LL-37
}

\author{
ZHONGSHUANG HU*, ISAO NAGAOKA* \\ * Department of Host Defense and Biochemical Research, Juntendo University Graduate School of Medicine, Tokyo, Japan
}

Pyroptosis is defined as a caspase- 1 dependent cell death and mainly occurs in macrophages and dendritic cells, accompanied with the release of pro-inflammatory cytokines. Moreover, pyroptosis results in the cellular lysis and release of cytosolic contents, which induce the augmentation of inflammatory reactions. Of note, caspase-1 knockout (KO) causes a protective effect on the sepsis models and improves the survival of mice, in which the IL-1 $\beta$ level was totally suppressed. Thus, caspase- 1 activation and possibly pyroptosis play a major role in the mortality of sepsis. AMPs (antimicrobial peptides) are functioning in the first line of defense against invading pathogens. Cathelicidin family of AMPs are recognized in many mammalian species, and LL-37 is the only one human cathelicidin. Besides its broad spectrum of antimicrobial actions, LL-37 has a role in innate immune defense, such as regulation of inflammatory responses and wound healing. Interestingly, LL-37 displays the protective effect on the endotoxemia models. In this review, we introduce a newly identified inhibitory effect of LL-37 on macrophage pyroptosis in vitro and in vivo.

Key words: antimicrobial peptide, LL-37, pyroptosis, macrophage, sepsis

\section{Introduction}

Sepsis is a life-threatening organ dysfunction induced by an uncontrolled host response to infection ${ }^{1)}$. In sepsis, over expression of inflammatory cytokines is a major mechanism for the multiple organ failure ${ }^{2)-4)}$. Recently, much attention has been focused on the mechanism for host cell death, which advances during sepsis and leads to the dysregulated inflammatory responses ${ }^{2) 5(6)}$.

Pyroptosis is a caspase- 1 dependent cell death of macrophages and dendritic cells recognized in bacterial infection ${ }^{7)}$. During pyroptosis, the cells promptly produce and release extracellularly the proinflammatory cytokines (such as $\mathrm{IL}-1 \beta)^{7)}{ }^{8}$. $\mathrm{IL}-1 \beta$ is a proinflammatory cytokine, which induces both local and systemic inflammatory/immune responses $^{9)}$, and acts cooperatively with other cytokines to cause tissue injury in sepsis $^{10}$. Pyroptosis is induced by the two distinct stimuli, microbial PAMPs (pathogen associated molecular patterns) (such as lipopolysaccharide/LPS) and endogenous DAMPs (damage associated molecular patterns) (such as ATP) (Figure-1) ${ }^{7)}{ }^{8)}$. TLRs (Toll like receptors) start a signaling that leads to cellular activation (including the upregulation of proinflammatory cytokines), in response to PAMPs ${ }^{8)}$. Furthermore, in responses to DAMPs, NLRs (Nod like receptors)/NLRPs (Nod like receptor proteins) are associated for the formation of inflammasome, where the active caspase-1is converted from the procaspase ${ }^{-1}{ }^{8}$. Lastly, IL-1 13 is processed and released by the activated caspase -1 , and cell death is induced ${ }^{8)}{ }^{11}$. Of note, caspase -1

Corresponding author: Zhongshuang $\mathrm{Hu}$

Department of Host Defense and Biochemical Research, Juntendo University Graduate School of Medicine

2-1-1 Hongo, Bunkyo-ku, Tokyo 113-8421, Japan

TEL: +81-3-5802-1033 FAX: +81-3-3813-3157 E-mail: chusou@juntendo.ac.jp

〔Received Mar. 10, 2016〕

Copyright (C) 2016 The Juntendo Medical Society. This is an open access article distributed under the terms of Creative Commons Attribution License (CC BY), which permits unrestricted use, distribution, and reproduction in any medium, provided the original source is properly credited. doi: $10.14789 / j m j .62 .98$ 


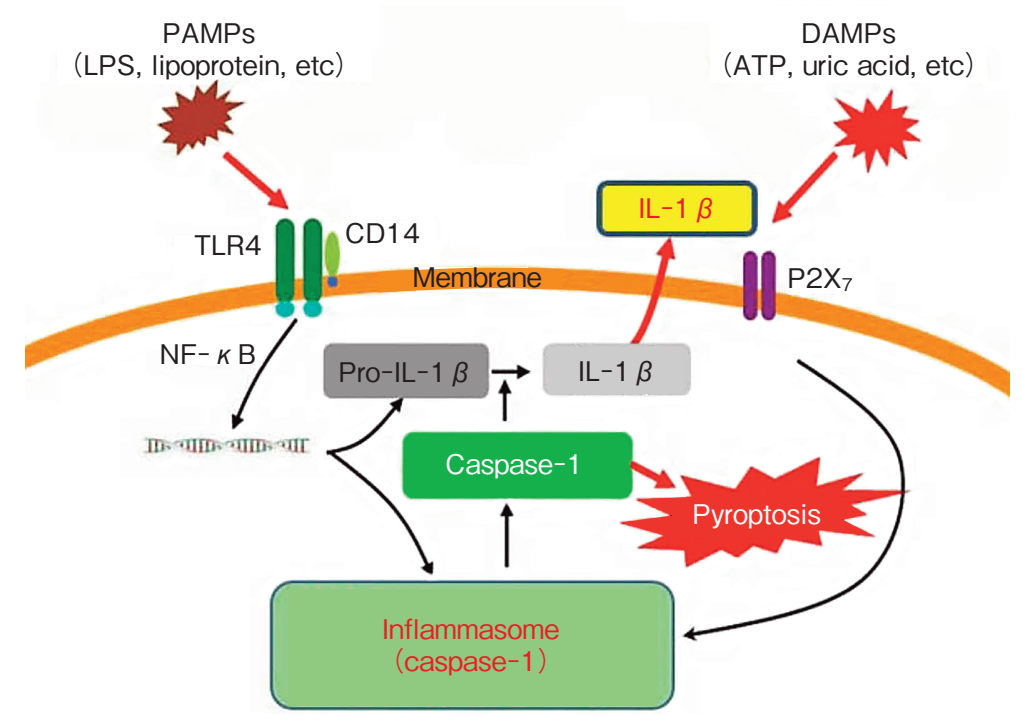

Figure-1 The stimulation of PAMPs and DAMPs induces pyroptosis

Pyroptosis is induced by the two distinct stimuli, microbial PAMPs (e.g. LPS and lipoproteins) and endogenous DAMPs (e.g. ATP and uric acid). In response to PAMPs, TLRs trigger a signaling leading to cellular activation (such as the upregulation of proinflammatory cytokines). In contrast, in responses to DAMPs, NLRs are associated for the formation of inflammasome, where the active caspase- 1 is converted for the procaspase- 1 . Finally, IL-1 $1 \beta$ is processed, and cell death is induced by the activated caspase- 1 .

knockout $(\mathrm{KO})$ causes a protective effect on several murine sepsis models and improves the survival of mice, where the IL-1ß level is completely depressed, and the organs (such as kidney and spleen) are protected $^{12)-16)}$. Thus, caspase- 1 activation and possibly pyroptosis play a major role in the pathogenesis (organ failure) and mortality of sepsis ${ }^{7)}$.

Antimicrobial peptides (AMPs), which are also called host defense peptides, function as the first line of defense against invading pathogens ${ }^{18}$. Cathelicidin family of AMPs are recognized in various mammalian species, and LL-37 is the only one human cathelicidin, which is cleaved from CAP18 (a cationic antimicrobial protein of $18-\mathrm{kDa}$ ) and primarily produced by neutrophils and epithelial cells ${ }^{18)}$. Besides its broad spectrum of bactericidal activities, LL-37 exhibits a wide range of inflammatory/immune modulatory activities ${ }^{18)}{ }^{19)}$. Of note, LL-37 suppresses TNF- $\alpha$ production by LPS-stimulated macrophages and improves the survival of LPS-induced endotoxin mice via the neutralization of LPS $^{20)}{ }^{21}$. Thus, as a therapeutic strategy in sepsis, LL-37 is attracting researchers' attention. Interestingly, LL-37 reduces the LPSinduced apoptosis of endothelial cells by neutralizing the action of LPS and inhibits the spontaneous apoptosis of neutrophils via the purinergic receptor
$\mathrm{X}_{7} \quad\left(\mathrm{P} 2 \mathrm{X}_{7}\right)$ and formyl-peptide receptor-like 1 (FPRL1) ${ }^{22)}$ 23), suggesting that LL-37 is involved in the modulation of cell death. Based on these facts, we focus on the effect of LL-37 on a macrophage cell death, pyroptosis in sepsis and introduce our results in this review.

\section{Inhibition of LPS/ATP-induced pyroptosis of macrophages by LL-37}

LPS is a major constituent of the outer membrane of gram-negative bacteria and function as a potent inducer of inflammatory responses by macrophages. Thus, LPS is a key molecule in the pathogenesis of gram-negative sepsis ${ }^{20)}{ }^{24)}$. LPS stimulates macrophages via the binding to the membrane receptors, CD14/TLR $4^{25)}$. In addition, extracellular ATP can be released from the dead host cells ${ }^{26)}$. For example, the plasma ATP level reaches to $10 \mu \mathrm{M}$ in the CLP (cecal ligation and puncture) septic mice $(2 \mu \mathrm{M} \text { in the Sham mice })^{27)}$, and the peritoneal ATP level increases up to $1 \mu \mathrm{M}$ in the $E$. coli-induced septic mice $(0.1 \mu \mathrm{M}$ in the Sham mice ${ }^{28)}$. Then, ATP activates a purinergic nucleotide receptor $\mathrm{P}_{2} \mathrm{X}_{7}$ to elicit the signal to induce the formation of inflammasome, where the active caspase- 1 is converted from the 


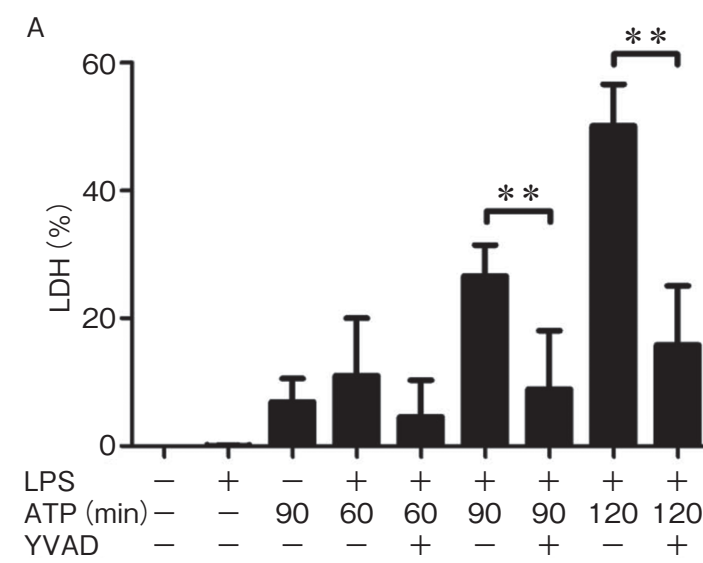

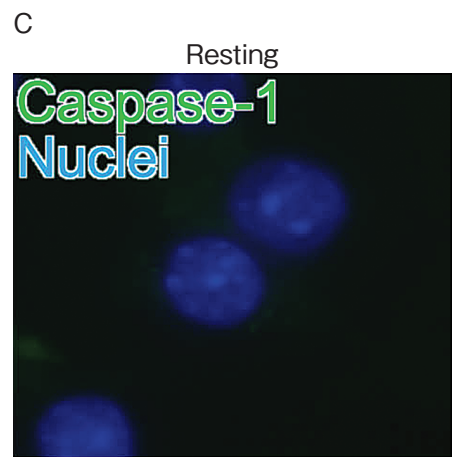

ATP

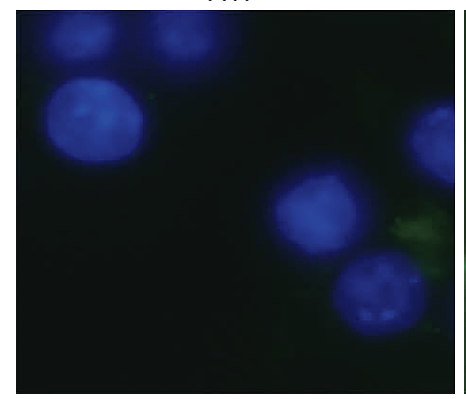

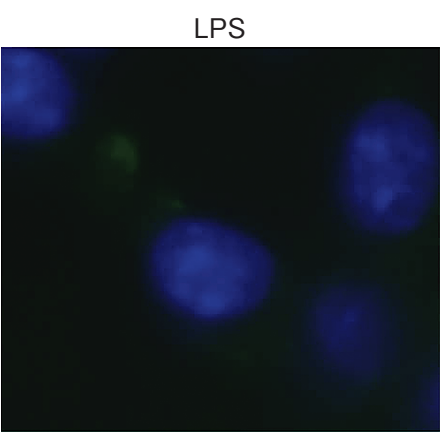

LPS/ATP

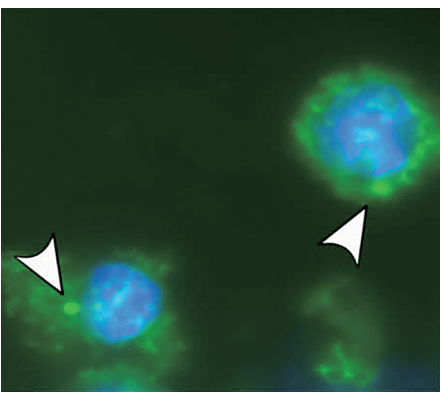

Figure-2 Effect of LPS and ATP on the pyroptosis of J774 cells

Macrophage-like cells (J774) were primed with $10 \mathrm{ng} / \mathrm{m} l$ LPS for $4 \mathrm{~h}$, and then stimulated with $3 \mathrm{mM}$ ATP for the indicated time intervals in the presence or absence of $20 \mu \mathrm{M} \mathrm{Ac}-\mathrm{YVAD}-\mathrm{CHO}$ (a caspase-1 specific inhibitor). J774 cells were also incubated without LPS and ATP (Resting) or with LPS or ATP alone. Then, the supernatants were assayed for IL-1B (A) and LDH (B). Data shows the mean \pm SD. Values are compared between the absence and presence of Ac-YVAD-CHO. ${ }^{*} *_{\mathrm{p}}<0.01,{ }^{*} *{ }^{*} \mathrm{p}<0.001$. C. J774 cells were primed with $10 \mathrm{ng} / \mathrm{m} l$ LPS for $4 \mathrm{~h}$ and then stimulated with $3 \mathrm{mM}$ ATP for $90 \mathrm{~min}$. Then, the cells were stained with FAM-YVAD-fmk (a fluorescent labeled caspase-1 inhibitor for inflammasome staining, green) and Hoechst 33342 (for nuclear staining, blue). Arrowheads indicate the inflammasomes, which contained the activated caspase -1 .

procaspase-1. Lastly, IL-1 $\beta$ is processed and released by the activated caspase- -1 , and cell death (pyroptosis) is induced (Figure-1) ${ }^{29)}$.

Thus, we use the LPS/ATP treatment as the typical inducers to assess the effect of LL-37 on macrophage pyroptosis in vitro. Mouse macrophage-like cells (J774) were primed with $10 \mathrm{ng} / \mathrm{ml}$ LPS, and then treated with ATP. LPS or ATP treatment alone did not essentially induce the production of IL-1 $1 \beta$ and cell death (as assessed by $\mathrm{LDH}$ release); however, the treatment with both LPS and ATP induced the production of IL- $1 \beta$ and cell death (Figure-2A and B). Of interest, $\mathrm{Ac}^{-}$ YVAD-CHO (a caspase- 1 specific inhibitor) suppressed both the LPS/ATP-induced release of

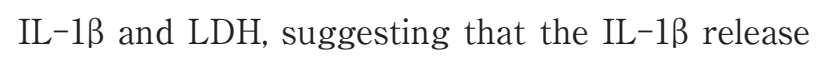

and cell death are caspase- 1 dependent. In fact, we confirmed by immunohistochemical staining that LPS/ATP induces the activation of caspase- 1 in inflammasome (Figure-2C). Moreover, the effect of LL-37 on the LPS/ATP-induced pyroptosis was investigated. Of importance, LL-37 substantially inhibited the LPS/ATP-induced IL-1B production, cell death (LDH release ${ }^{30}{ }^{30}$. In addition, we confirmed by flow cytometry that LPS/ATP induces the caspase -1 activation, and LL-37 significantly suppresses the LPS/ATP-induced caspase-1 activation $^{30)}$.

To clarify the mechanism for the inhibitory action of LL-37, we assessed the action of LL-37 on the LPS binding to the cells. LL-37 potently inhibited the binding of LPS to its receptor CD14/TLR4 on 
J774 cells ${ }^{30}$. Together these observations apparently indicate that LL-37 suppresses the LPS/ ATP-induced pyroptosis via the inhibition of the LPS binding to its receptor (CD14/TLR4). Moreover, we confirmed that ATP stimulates the nucleotide receptor $\left(\mathrm{P}_{2} \mathrm{X}_{7}\right)$ to activate caspase -1 in J774 cells and the activation was suppressed by the $\mathrm{P}^{2} \mathrm{X}_{7}$ antagonists (KN-62 and $\left.\mathrm{KN}-93\right)^{30)}$. Importantly, LL-37 substantially inhibited the ATPinduced caspase- 1 activation ${ }^{30}$, indicating that LL-37 clearly inhibits the ATP-induced P2X activation.

Together these observations indicate that the treatment with LPS/ATP induces the pyroptosis of macrophage-like cells, and that LL-37 suppresses the LPS/ATP-induced pyroptosis, which is associated with $\mathrm{IL}-1 \beta$ release, caspase -1 activation and cell death. In addition, LL-37 represses the LPS binding to the cells, and the ATP-induced $\mathrm{P}_{2} \mathrm{X}_{7}$ activation, indicating that LL-37 inhibits the pyroptosis via both the neutralization of LPS action and the inhibition of the $\mathrm{P}_{2} \mathrm{X}_{7}$ response to ATP (Figure-5, left panel).

\section{Inhibition of the macrophage pyroptosis and} improvement of the survival of septic mice by LL-37

Based on the above findings, we hypothesize that LL-37 may inhibit the pyroptosis of macrophages in vivo, thereby exhibiting the protective action on septic model. Thus, we intravenously administered LL-37 into a CLP septic model of mice, and evaluated the effect of LL-37 on pyroptosis by analyzing caspase -1 activation, cell death and IL-1 release.

First, we evaluated the effect of LL-37 administration on the survival of CLP-operated mice. Interestingly, the administration of LL-37 $(2 \mu \mathrm{g} /$ mouse) significantly improved the survival rate (Figure-3). Next, to evaluate the effect of LL-37 on pyroptosis of macrophages, peritoneal cells were harvested at $5 \mathrm{~h}$ after the CLP operation, and peritoneal macrophages were evaluated for pyroptosis by detecting caspase- 1 activation and cell death (7AAD positive). As shown in Figure-4, caspase- 1 activation was significantly higher in the CLP group than that in the Sham group. Similarly, pyroptosis (caspase-1-activated dead cells) was obviously induced in the CLP group. Of note, the

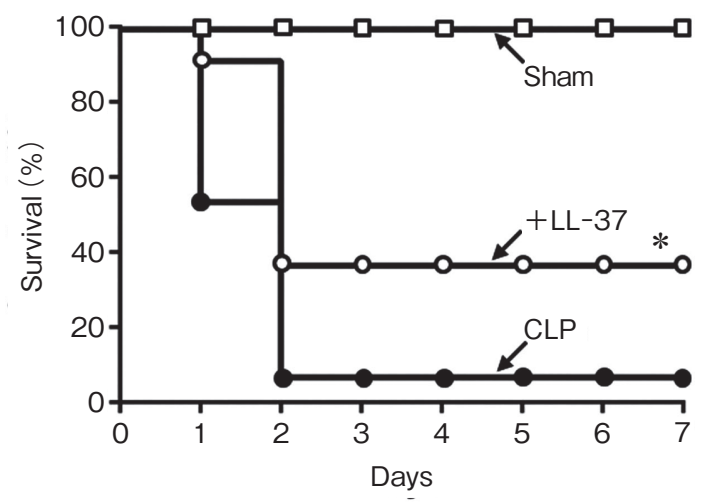

Figure-3 Effect of LL-37 on the survival of CLP septic mice

Mice were divided into the Sham $(\square), \operatorname{CLP}(\bullet)$ and LL-37 (○) groups. In the LL-37 group, mice were intravenously administered with $2 \mu \mathrm{g}$ per mouse LL-37, and the survival rates of the mice were monitored for 7 days. Survival data were analyzed using the Kaplan-Meier method and survival curves were compared using the log-rank test and Gehan-Breslow-Wilcoxon test in univariate analysis. ${ }^{*} \mathrm{p}<0.05$.

administration of LL-37 significantly suppressed the CLP-induced caspase -1 activation and pyroptosis of peritoneal macrophages (Figure-4B and C). Moreover, LL-37 modulated the levels of inflammatory cytokines (IL-1 $\beta$, IL- 6 and TNF- $\alpha$ ) both in the peritoneal fluids and sera and suppressed the activation of peritoneal macrophages (as evaluated by the increase in the intracellular levels of IL-1B, IL- 6 and TNF- $\alpha{ }^{31}{ }^{31}$. Furthermore, LL-37 reduced the bacterial burdens both in peritoneal fluids and blood samples ${ }^{31}$. Thus, our present study suggests that LL-37 improves the survival of CLP septic mice by possibly suppressing the pyroptosis of macrophages, and inflammatory cytokine production by activated macrophages as well as bacterial growth (Figure-5, right panel).

Importantly, as reported above, the combination of LPS and ATP induced the pyroptosis of macrophages, which was inhibited by LL-37 via both the neutralization of LPS action and inhibition of the $\mathrm{P}_{7} \mathrm{X}_{7}$ response to ATP. Since LPS and ATP are major inducer of caspase- 1 activation and pyroptosis in the CLP septic mice in various septic models ${ }^{32}{ }^{33)}$, we speculate that LL-37 suppresses the actions of LPS and ATP in vivo as well as in vitro, thereby inhibiting pyroptosis (caspase-1 activation) and increasing the survival of CLP septic mice. 
A

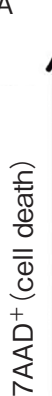
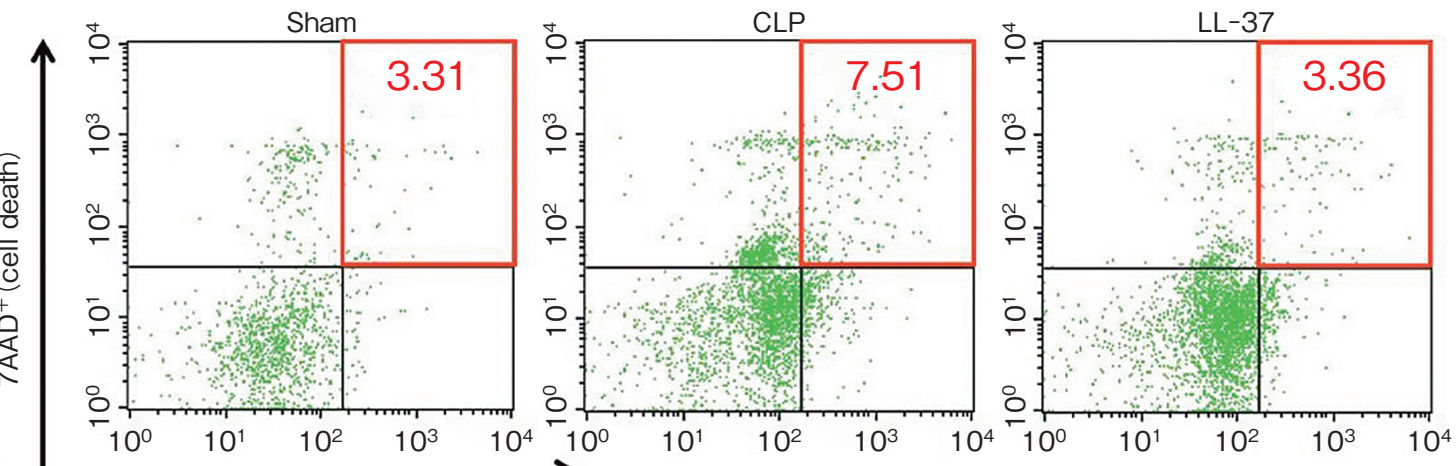

$\mathrm{FLICA}^{+}$(caspase-1 activation)

B

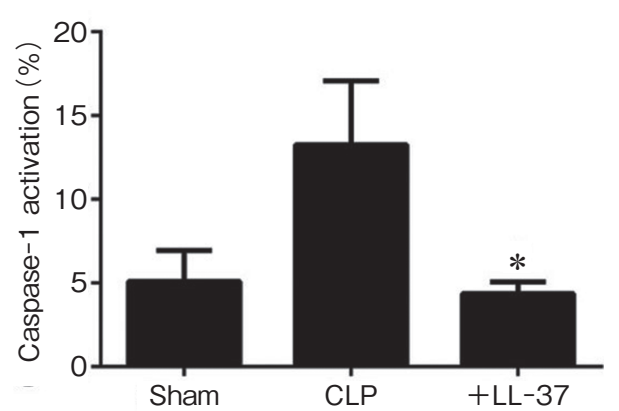

C

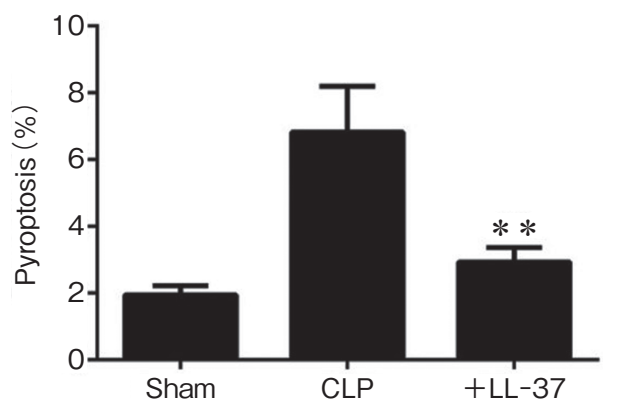

Figure-4 Effect of LL-37 on the pyroptosis of peritoneal macrophages

Peritoneal cells were collected from mice of Sham, CLP and LL-37 groups at $5 \mathrm{~h}$ after the surgery. Thereafter, peritoneal cells were evaluated for pyroptosis by detecting caspase- 1 activation (FLICA positive) and cell death (7AAD positive) of the peritoneal macrophages (F4/80 positive). In panel A, upper halves, right halves and upper-right quadrants show cell death, caspase-1 activation and pyroptosis (FLICA/7AAD-double positive), respectively, among peritoneal macrophages. Images are representative of 6-7 separate experiments. Panels $B$ and $C$ show the percentage of caspase -1 activation and pyroptosis, respectively. Data are the mean \pm SEM, and values are compared between the CLP and LL-37 groups. ${ }^{*} \mathrm{p}<0.05,{ }^{*}{ }^{*} \mathrm{p}<0.01$.
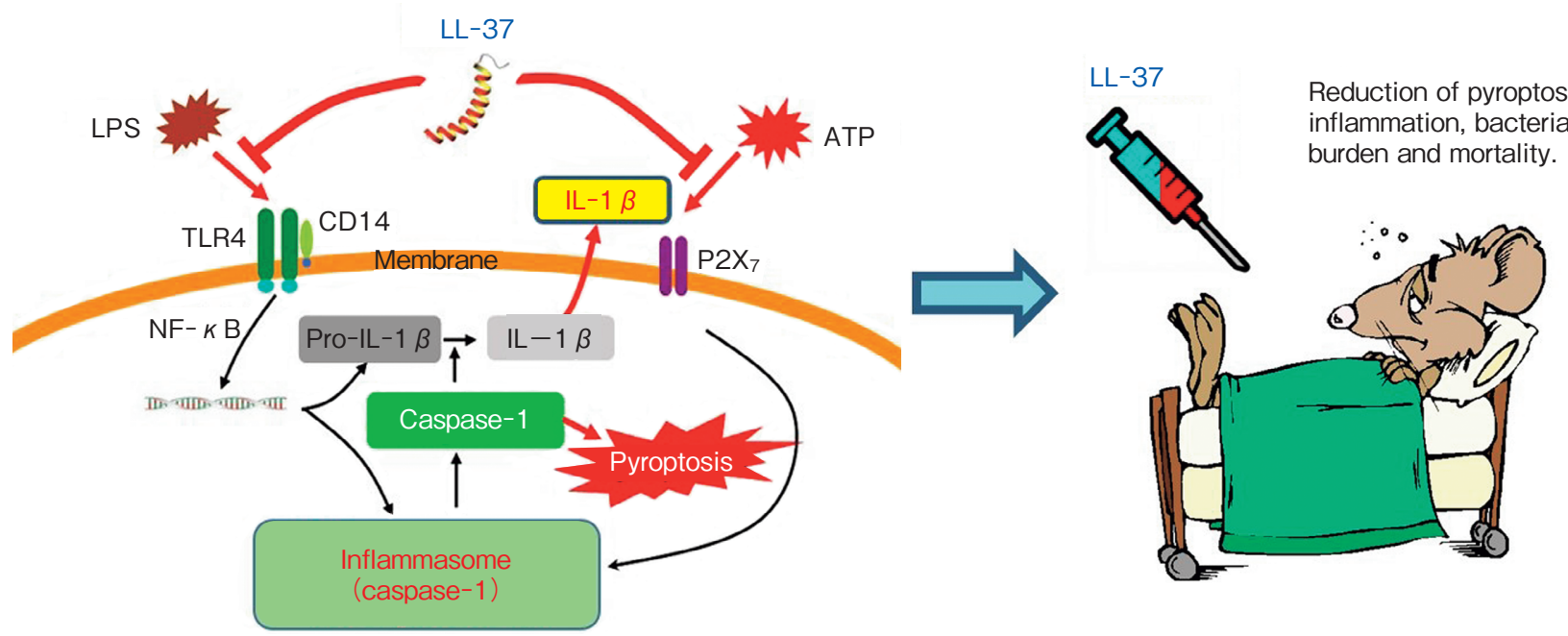

Figure-5 Effect of LL-37 on pyroptosis of macrophages and sepsis

LPS and ATP treatment induces the pyroptosis of macrophages, and LL-37 inhibits the LPS/ATP-induced pyroptosis (associated with IL- $1 \beta$ release, caspase -1 activation and cell death), by both the neutralization of LPS action and the inhibition of the P2X $\mathrm{X}_{7}$ response to ATP (left panel). The administration of LL-37 reduces the pyroptosis of macrophages, inflammatory cytokine production and bacterial growth, and improves the survival of CLP septic mice (right panel). 


\section{Conclusion}

Pyroptosis is recently identified as a caspase- 1 dependent cell death of macrophages and dendritic cells observed in bacterial infection ${ }^{7)}$. During pyroptosis, the cells promptly produce and release extracellularly IL-1 $1 \beta$, which acts cooperatively with other cytokines to trigger tissue injury in sepsis $^{7)-10)}$. Of note, caspase- 1 KO causes a protective effect on several murine sepsis models and

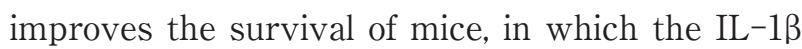
level was totally reduced, and the organs (such as kidney and spleen) were protected ${ }^{12)-16)}$. Thus, caspase -1 activation and possibly pyroptosis play a major role in the pathogenesis (organ failure) and mortality of sepsis.

LL-37 is initially identified as an $\mathrm{AMP}^{18)}$, and participates in the innate immune system, with the abilities to protect the host from invasive microbial infections and neutralize gram-negative LPS, and now recognized as a multifunctional molecule linking the innate immune response to the adaptive immune system via the diverse immunomodulatory actions (such as induction of the cytokine and chemokine production, the immune cell chemotaxis, and the alteration of dendritic cell differentiation) ${ }^{18)-21)}$. In this review, we demonstrated an in vitro function of LL-37 to suppress the pyroptosis of macrophages via the neutralization of LPS action and the inhibition of $\mathrm{P}_{2} \mathrm{X}_{7}$ response and an in vivo function of LL-37 to suppress the caspase-1 activation and pyroptosis of macrophages, using a polybacterial CLP sepsis model. As a therapeutic strategy, the blocking of LPS action (e.g. TLR4 KO) protects mice from systemic inflammation and tissue damage in endotoxemia ${ }^{34)}$, and the blocking of ATP action (e. g. administration of a P2X antagonist) improve the survival rate in $E$. coliinduced sepsis ${ }^{35}$. Thus, the present findings indicate that $\mathrm{LL}-37$ can be a promising candidate for sepsis because it has potent biological functions, including the inhibition of pyroptosis, modulation of inflammatory cytokine production and antimicrobial activity (Figure-5).

\section{References}

1) Singer M, Deutschman CS, Seymour CW, et al: The third international consensus definitions for sepsis and septic shock (Sepsis-3). JAMA, 2016; 315: 801-810.

2) Pinsky MR: Dysregulation of the immune response in severe sepsis. Am J Med Sci, 2004; 328: 220-229.

3) Matsuda A, Jacob A, Wu R, et al: Novel therapeutic targets for sepsis: regulation of exaggerated inflammatory responses. J Nippon Med Sch, 2012; 79: 4-18.

4) Netea MG, van der Meer JW, van Deuren M, et al: Proinflammatory cytokines and sepsis syndrome: not enough, or too much of a good thing? Trends Immunol, 2003; 24: 254-258.

5) Wesche DE, Lomas-Neira JL, Perl M, et al: Leukocyte apoptosis and its significance in sepsis and shock. J Leukoc Biol, 2005; 78: 325-337.

6) Pinheiro da Silva F, Nizet V: Cell death during sepsis: integration of disintegration in the inflammatory response to overwhelming infection. Apoptosis, 2009; 14: 509-521.

7) Miao EA, Rajan JV, Aderem A: Caspase-1-induced pyroptotic cell death. Immunol Rev, 2011; 243: 206-214.

8) Bergsbaken T, Fink SL, Cookson BT: Pyroptosis: host cell death and inflammation. Nat Rev Microbiol, 2009; 7: 99-109.

9) Gabay C, Lamacchia C, Palmer G: IL-1 pathways in inflammation and human diseases. Nat Rev Rheumatol, 2010; 6: 232-241.

10) Casey LC, Balk RA, Bone RC: Plasma cytokine and endotoxin levels correlate with survival in patients with the sepsis syndrome. Ann Intern Med, 1993; 119: 771-778.

11) Latz E: The inflammasomes: mechanisms of activation and function. Curr Opin Immunol, 2010; 22: 28-33.

12) Sarkar A, Hall MW, Exline M, et al: Caspase-1 regulates Escherichia coli sepsis and splenic B cell apoptosis independently of interleukin-1beta and interleukin-18. Am J Respir Crit Care Med, 2006; 174: 1003-1010.

13) Li P, Allen H, Banerjee S, et al: Mice deficient in IL-1 beta-converting enzyme are defective in production of mature IL-1 beta and resistant to endotoxic shock. Cell, 1995; 80: 401-411.

14) Vanden Berghe T, Demon D, Bogaert P, et al: Simultaneous targeting of IL-1 and IL-18 is required for protection against inflammatory and septic shock. Am J Respir Crit Care Med, 2014; 189: 282-291.

15) Lamkanfi M, Sarkar A, Vande Walle L, et al: Inflammasome-dependent release of the alarmin HMGB1 in endotoxemia. J Immunol, 2010; 185: 4385-4392.

16) Wang W, Faubel S, Ljubanovic D, et al: Endotoxemic acute renal failure is attenuated in caspase-1-deficient mice. Am J Physiol Renal Physiol, 2005; 288: 997-1004.

17) Croker BA, O'Donnell JA, Gerlic M: Pyroptotic death storms and cytopenia. Curr Opin Immunol, 2014; 26: 128-137.

18) Durr UH, Sudheendra US, Ramamoorthy A: LL-37, the only human member of the cathelicidin family of antimicrobial peptides. Biochim Biophys Acta, 2006; 1758: $1408-1425$.

19) Hancock RE, Diamond G: The role of cationic antimicrobial peptides in innate host defences. Trends Microbiol, 2000; 8: 402-410.

20) Nagaoka I, Hirota S, Niyonsaba F, et al: Cathelicidin family of antibacterial peptides CAP18 and CAP11 inhibit the expression of TNF-alpha by blocking the binding of LPS to CD14 (+) cells. J Immunol, 2001; 167: 3329-3338.

21) Nagaoka I, Hirota S, Niyonsaba F, et al: Augmentation of 
the lipopolysaccharide-neutralizing activities of human cathelicidin CAP18/LL-37-derived antimicrobial peptides by replacement with hydrophobic and cationic amino acid residues. Clin Diagn Lab Immunol, 2002; 9: 972-982.

22) Suzuki K, Murakami T, Kuwahara-Arai K, et al: Human anti-microbial cathelicidin peptide LL-37 suppresses the LPS-induced apoptosis of endothelial cells. Int Immunol, 2011; 23: 185-193.

23) Nagaoka I, Tamura H, Hirata M: An antimicrobial cathelicidin peptide, human CAP18/LL-37, suppresses neutrophil apoptosis via the activation of formyl-peptide receptor-like 1 and P2X7. J Immunol, 2006; 176: 3044-3052.

24) Fan H, Cook JA: Molecular mechanisms of endotoxin tolerance. J Endotoxin Res, 2004; 10: 71-84

25) Triantafilou M, Triantafilou K: Lipopolysaccharide recognition: CD14, TLRs and the LPS-activation cluster. Trends Immunol, 2002; 23: 301-304.

26) Trautmann A: Extracellular ATP in the immune system: more than just a "danger signal". Sci Signal, 2009; 2: pe6.

27) Sumi Y, Woehrle T, Chen Y, et al: Plasma ATP is required for neutrophil activation in a mouse sepsis model. Shock, 2014; 42: 142-147.

28) Xiang Y, Wang X, Yan C, et al: Adenosine-5'-triphos- phate (ATP) protects mice against bacterial infection by activation of the NLRP3 inflammasome. PLoS One, 2013; 8: e63759.

29) Bryant C, Fitzgerald KA: Molecular mechanisms involved in inflammasome activation. Trends Cell Biol, 2009; 19: 455-464.

30) $\mathrm{Hu} \mathrm{Z}$, Murakami T, Suzuki K, et al: Antimicrobial cathelicidin peptide LL-37 inhibits the LPS/ATPinduced pyroptosis of macrophages by dual mechanism. PLoS One, 2014; 9: e85765.

31) $\mathrm{Hu} \mathrm{Z}$, Murakami T, Suzuki K, et al: Antimicrobial cathelicidin peptide LL-37 inhibits the pyroptosis of macrophages and improves the survival of polybacterial septic mice. Int Immunol, 2016 (in press)

32) Griffiths RJ, Stam EJ, Downs JT, et al: ATP induces the release of IL-1 from LPS-primed cells in vivo. J Immunol, 1995; 154: 2821-2828.

33) Cauwels A, Rogge E, Vandendriessche B, et al: Extracellular ATP drives systemic inflammation, tissue damage and mortality. Cell Death Dis, 2014; 5: e1102.

34) Wittebole $\mathrm{X}$, Castanares-Zapatero D, Laterre PF: Toll-like receptor 4 modulation as a strategy to treat sepsis. Mediators Inflamm, 2010; 2010: 568396.

35) Wewers MD, Sarkar A: P2X 7 receptor and macrophage function. Purinergic Signal, 2009; 5: 189-195. 